\title{
Super-Resolution and Large Depth of Field Model for Optical Microscope Imaging
}

\author{
Ruo-Peng Zheng, Shu-Bin Liu, and Lei Li \\ School of Electronics and Information Engineering, Sichuan University, Chengdu 610065, China \\ Correspondence should be addressed to Lei Li; leili@scu.edu.cn
}

Received 24 November 2021; Accepted 11 December 2021; Published 29 December 2021

Academic Editor: Paramasivam Senthilkumaran

Copyright ( 2021 Ruo-Peng Zheng et al. This is an open access article distributed under the Creative Commons Attribution License, which permits unrestricted use, distribution, and reproduction in any medium, provided the original work is properly cited.

\begin{abstract}
Due to the limitation of numerical aperture (NA) in a microscope, it is very difficult to obtain a clear image of the specimen with a large depth of field (DOF). We propose a deep learning network model to simultaneously improve the imaging resolution and DOF of optical microscopes. The proposed M-Deblurgan consists of three parts: (i) a deblurring module equipped with an encoder-decoder network for feature extraction, (ii) an optimal approximation module to reduce the error propagation between the two tasks, and (iii) an SR module to super-resolve the image from the output of the optimal approximation module. The experimental results show that the proposed network model reaches the optimal result. The peak signal-to-noise ratio (PSNR) of the method can reach 37.5326, and the structural similarity (SSIM) can reach 0.9551 in the experimental dataset. The method can also be used in other potential applications, such as microscopes, mobile cameras, and telescopes.
\end{abstract}

\section{Introduction}

In microscopy, it is very difficult to obtain a clear image of the specimen with a large depth of field (DOF), especially for large NA. Usually, optical axial scanning is used to solve the problem. However, it is time-consuming. With the rapid development of deep learning in computer vision and image processing, it is possible to use deep learning algorithms to obtain a clear image of the specimen with a large DOF. For example, the super-resolution (SR) and deblurring algorithms are used to improve the resolution of images [1-9]. Recently, the deep learning single-image super-resolution (SISR) model [10-12] and convolutional neural network (CNN) model [13] have been applied to improve the resolution and definition in microscope images, which indicates that they have great potential in microscopes. However, compared with the application of SISR in enhancing the texture of real images, the SR microscopic images have higher requirements for the predicted image fineness. Therefore, it is still an uncertain problem that the SR image based on deep learning is better than the traditional SR microscope [14-16] in many scenarios. Public image deblurring methods in deep learning simply solve the blur caused by the point-spread function (PSF) of the optical microscope [17], while the problem of the blurred microscope images due to insufficient DOF needs to be solved urgently. For example, traditional SR microscopes such as localization microscopy [14], STED microscopy [15], and structured illumination microscopy (SIM) [16] provide great convenience to observe the internal work of cells and various biological processes. However, it needs a relatively more complicated experimental setup and requires a priori knowledge about the sample and its preparation. An SR confocal microscope based on deep learning is proposed to improve the resolution of the image [18], but this method is based on photoactivated localization microscopy (PALM), and it is difficult to generalize it in various microscopic imaging systems. An SR microscope based on generative adversarial networks (GAN) enhances the textures of macroscale realistic photographs, but the fineness is far from the requirements of microscopic imaging [19]. An SR microscope based on deep neural networks has greater accuracy and quantifiability of the inferred nanoscale structure [20]. But the blur inherent in low-resolution (LR) images is 
still unresolved. A deblur microscope based on CNN is proposed to remove the blur caused by PSF [17]. This method ignores the blur caused by the insufficient DOF. Therefore, it is urgent to realize to study a microscope with both SR and large DOF functions.

In this paper, we propose a generative adversarial neural network framework model (M-Deblurgan) based on the optimal approximation, which is to simultaneously solve the low-resolution and insufficient depth of field in an optical microscope. M-Deblurgan is composed of a generator network and a discriminator network that have a competitive relationship. The generator network comprises three modules: deblurring module, optimal approximation module, and SR module. The robustness of the model on the publicly available dataset and experimental dataset are tested. The PSNR of the method can reach 37.5326, and the SSIM can reach 0.9551 in the experimental dataset, which shows the deblurred results and resolution are improved compared to other models.

\section{Proposed Method}

The proposed method is shown in Figure 1. The proposed deep learning network structure follows the GAN framework [21], which has two subnetworks trained at the same time. One is a generator network that makes LR microscopic blurred images become high-resolution clear microscopic images, and the other is a discriminator network that distinguishes the image generated by the generator from the real image.

2.1. Network Structure. The generator network is shown in Figure 1(a). We integrate the ideas of residual network and encoder-decoder network into the joint task of microscopic image deblurring and SR. The generator network consists of a deblurring module, an optimal approximation module, and an SR module.

In the deblurring module, we propose an improved encoder-decoder network as our deblurring module and use the improved method as a lightweight option to incorporate multiscale features. The architecture consists of an encoderdecoder network, and we output the final feature maps of five different scales during the upsampling process. The encoder is made up of a $5 \times 5$ flat-convolutional layer and four $3 \times 3$ Res-Blocks. To broaden the perceptual field and learn high-dimensional representations, the encoder component employs three pooling layers. Three $3 \times 3$ upsampling layers and three $3 \times 3$ Res-Blocks make up the decoder component, which gradually increases the size of the feature map to the original input resolution. Between the encoder and the decoder, we added four skip connections. These connections can hasten the network's convergence and result in significantly crisper outputs. To avoid adding too many fuzzy features from the encoder side, we additionally use a $3 \times 3$ flat-convolution layer before element-wise addition. These feature maps of different scales are sampled to the same 1/4 input size and connected into a tensor that contains rich image texture information of different scales.
There are two upsampling and convolutional layers at the end of the network, which can make the output size of the deblurring module equal to the input size and reduce artifacts. We have also made some improvements to the residual block. We removed the batch normalization layer in ResBlock compared to the original ResNet. The batch normalization layer normalizes the properties of specific jobs, increases the amount of computation, and lowers the flexibility of the network.

In the optimal approximation module, firstly, this module can avoid the accumulation of errors due to the simple series connection of the deblurring module and the SR module, that is, to prevent the estimation error of the first model from being further propagated and amplified in the second model. Secondly, it makes full use of the correlation between the two tasks. The main formulas used in our optimal approximation method are as follows:

$$
F\left(x_{i}\right)=\frac{1}{e^{\left|x_{i}-z\right|}} .
$$

Among them,

$$
Z=\frac{\left\|\left(x_{1}, \ldots, x_{i}, \ldots, x_{N}\right)\right\|_{2}}{N} .
$$

$N$ in equation (2) represents the number of inputs, and $x_{i}$ represents the $\mathrm{i}$-th input. $\mathrm{N}$ in this network is 3 . $\left(x_{1}, \ldots, x_{i}, \ldots, x_{N}\right)$ represents the set of inputs, which represents the input image, the output of the encoder-decoder network, and the output of the deblurring module in the proposed network, respectively. The two formulas are mainly used to determine which of the 3 inputs is closest to the actual optimal input. The input picture and the output of the feature module are connected to the optimal approximation module in a jump connection. Through the screening of the optimal approximation module, the data of the one closest to the real picture can be output. The forward and backward jump connections can minimize the influence of the front network error on the backpropagation and reduce the network coupling.

In the super-resolution module, the output of the optimal approximation module is used as the input of the SR module. The model has three fully connected layers (FC) with a core size of 3 . Then, the feature map resolution is improved by two trainable 2x subpixel convolutional layers. The model adds a convolutional layer with a core size of 3 at the tail to convert the features to the RGB image and remove redundant artifacts. We add residual learning to the superresolution module and add a global jump connection to jointly reconstruct the super-resolution image, making the final image closer to the real image.

The input of the discriminator is composed of the image generated by the generator and the real image, and the output is the probability that the image generated by the generator is the real image. The network structure is shown in Figure 1(b). The network is similar to the VGG network, which uses step-size convolution to downsample the image while doubling the number of feature maps. Except for the sigmoid function used in the last layer, the Leaky ReLU (LR) 


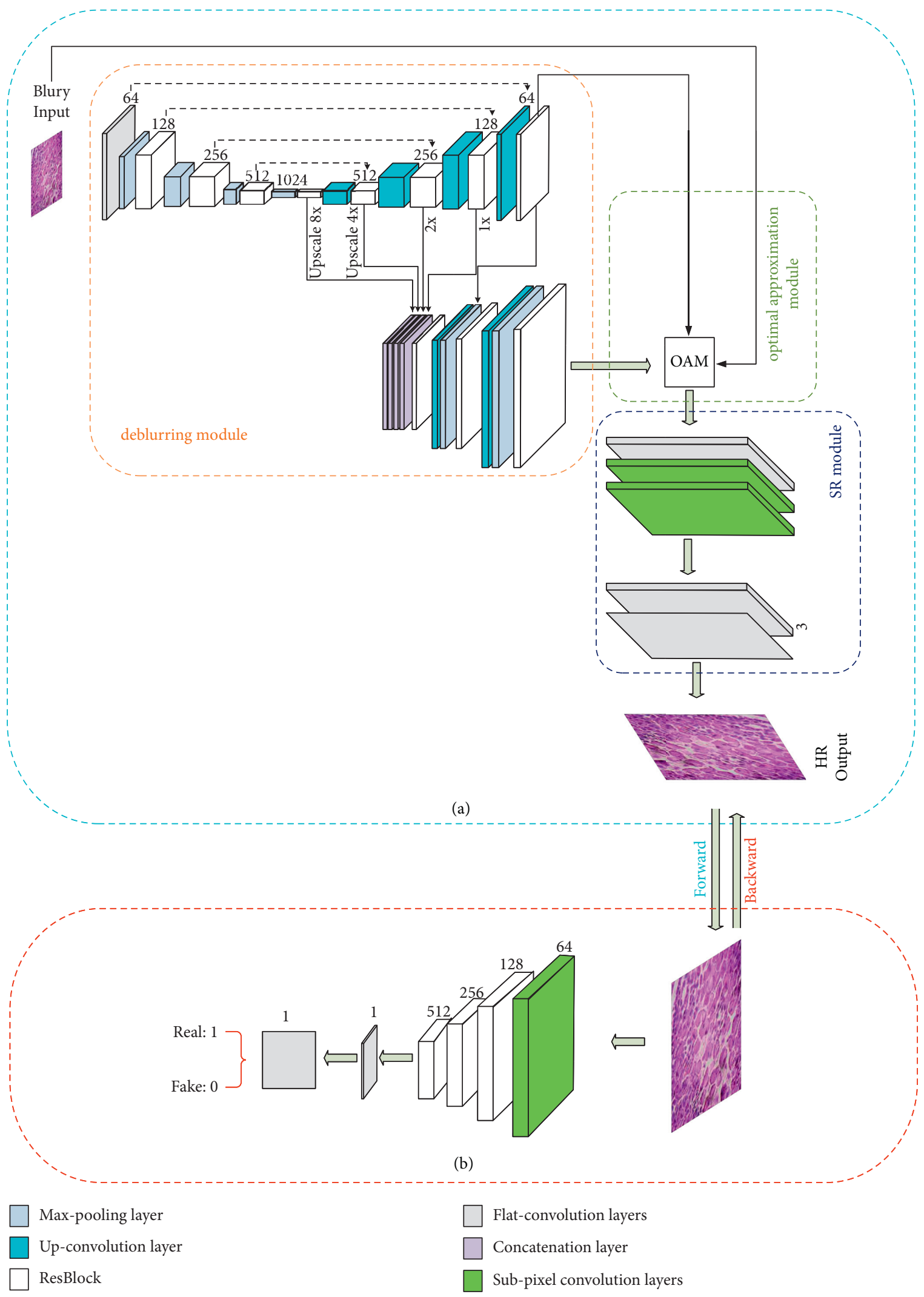

FIgURE 1: M-Deblurgan pipeline architecture. (a) Generator network. (b) Discriminator network. 
activation function with a slope of 0.2 is used after the other layers. Besides, we use a global average pooling layer to replace the first fully connected layer, which can prevent overfitting and reduce the number of parameters in the model training process.

2.2. Network Loss. The designed objective function is the combination of three different loss functions in M-Deblurgan: pixel-wise mean squared error (MSE) loss $\left(L_{\mathrm{MSE}}\right)$, perceptual loss $\left(L_{\text {percep }}\right)$, and SSIM loss $\left(L_{\mathrm{SSIM}}\right)$. In the proposed method, the MSE loss is calculated as follows:

$$
L_{\mathrm{MSE}}=\frac{1}{N} \sum_{i=1}^{N}\left(\left\|G_{1}\left(F\left(I_{i}^{L R}\right)\right)-I_{i}^{L k}\right\|^{2}+\left\|G_{2}\left(I_{i}^{D}\right)-I_{i}^{\mathrm{HR}}\right\|^{2}\right) \text {. }
$$

Among them, $I_{i}^{\mathrm{LR}} I_{i}^{\mathrm{LR}}, I_{i}^{D}$, and $I_{i}^{\mathrm{HR}}$ represent LR microscopically blurred images, LR clear images, deblurred images, and high-resolution clear images, respectively. $F$ represents the feature extraction module, $G_{1}$ represents the deblurring module, and $G_{2}$ represents the SR module. The perceptual loss is calculated as follows:

$$
L_{\text {percep }}=\frac{1}{W_{i, j} H_{i, j}} \sum_{x=1}^{W_{i, j}} \sum_{y=1}^{H_{i, j}}\left(\varphi_{i, j}\left(I^{\mathrm{HR}}\right)_{x, y}-\varphi_{i, j}\left(G\left(I^{\mathrm{LR}}\right)\right)_{x, y}\right)^{2} .
$$

Among them, $\phi_{i, j}$ represents the feature map output in the $\mathrm{i}$-th pooling layer, and the $\mathrm{j}$-th convolutional layer after the image is input to the pretrained VGG19 network model. $W_{i, j}$ and $H_{i, j}$ represent the dimension of the feature map. Specifically, we aim to minimize from the following equation:

$$
\begin{aligned}
L(G ; D)= & L_{\mathrm{MSE}}+\alpha\left(L_{\text {percep }}\right) \\
& -\beta \log \left|\frac{\left(1+\operatorname{SSIM}\left(G\left(I^{\mathrm{LR}}\right), y\right)\right)}{2}\right|,
\end{aligned}
$$

where $G\left(I^{\mathrm{LR}}\right)$ is the generative model output, $D($.$) is the$ predicted result of the discriminator, and $y$ is the HR image used as ground truth. Since the images generated by the model often have unsatisfactory artifacts and excessively smooth textures, $\alpha$ and $\beta$ were set to accommodate the MSE loss and the SSIM loss to be $\sim 5-15 \%$ of the combined generator model loss $L(G ; D)$.

\section{Experimental Results and Analysis}

The proposed model is trained and tested on a PC (Intel Core i7-10750H CPU @2.6 GHz+RTX1660Ti) equipped with the Windows 10 operating system and is based on the platform of PyTorch 1.7.0. We set the learning rate to $10^{-4}$ in the first 150 epochs and linearly decayed to $10^{-7}$ in the subsequent 50 epochs.

The FIRE (https://www.ics.forth.gr/cvrl/fire/) dataset is used as the simulated dataset for the training and testing of the proposed network. We cropped the dataset into 5360 images with a size of $512 \times 512$, of which 4840 images are used for training and 520 images are used for testing. For training, the training dataset is down-sampled 4 times through bicubic interpolation to obtain an LR image with a spatial resolution of $256 \times 256$. Finally, the blurry LR inputs are generated by applying a Gaussian kernel. The proposed network model processes a $256 \times 256$ blurry LR input and outputs an SR and a clear image of $512 \times 512$ size. The results of the experiment are shown in Figure 2. Figure 2(a) is an original uncropped image. Figures 2(b), 2(d), and 2(f) represent the enlarged images of the red frame region enclosed in Figure 2(a). Figures 2(c), 2(e), and 2(g) represent the enlarged images of the blue frame region enclosed in Figure 2(a). Figures 2(b) and 2(c) are blurry LR inputs. By using Figures 2(b) and 2(c) as the model input, the output is the clear SR images shown in Figures 2(d) and 2(e). From the experimental results, Figures 2(d) and 2(e) remove the blur of small blood vessels and improve the resolution of the image (as indicated by the red and blue arrows). The restored image is very close to the ground truth (Figures 2(f) and $2(\mathrm{~g}))$ in terms of details and intensity information.

Our model performs very well in the simulated dataset. It is very necessary to use the PINE CONES dataset collected in a real and complex experimental environment to verify the quality of the model. The experimental image acquisition and processing process is shown in Figure 3.

Microscopic defocused images of female pine cones are obtained by the $\times 5$ objective lens of a biological microscope. At the same time, the clear images of the corresponding $\times 10$ objective are obtained as the ground truth for model training. The experimental observation device is shown in Figure 4 . The specimen is the female pine cones. We train and test our proposed model and other SR and deblurring methods on the PINE CONES dataset. The results are shown in Figure 5. Figure 5(a) is obtained by the $\times 5$ objective with the process of the defocused blur. Figures $5(\mathrm{~b})-5(\mathrm{~d})$ are the images obtained by processing Figure 5(a) with the RLDecov + SR method [22], the U-net + SR method [23], and our method, respectively. Figure $5(\mathrm{e})$ is obtained by the $\times 10$ objective. Figures $5(\mathrm{f})$ and $5(\mathrm{j})$ and $5(\mathrm{k})-5(\mathrm{o})$ are the enlarged images of two same areas in Figures 5(a)-5(e), respectively. These areas are pointed out in Figures 5(a)-5(e) using solid red and blue frames. It can be seen from the enlarged image Figures 5(f)-5(o) that there are serious distortions in the restoration of blurry LR images through the RL-Decov + SR and U-net + SR methods. The cell image recovered by the RL-Decov $+\mathrm{SR}$ method is shown in Figures 5(g) and 5(l). It is not difficult to find that the nucleus and the cell wall are diffused. The cell image recovered by the $\mathrm{U}$-net + SR method is shown in Figure 5(h) with unsatisfactory artifacts, and the sharpening of the cell wall is not enough in Figure 5(m). However, the processed image in Figure 5(d), obtained using our method, is similar to Figure 5(e).

The model proposed in this paper reconstructs a clear and high-resolution image when the input is a low-resolution microscopic blurred image while maintaining a small model size. In the prediction phase, the results can be output quickly. When reconstructing the image, only the generator network model is required. Since the generator of the proposed method belongs to a fully convolutional neural 

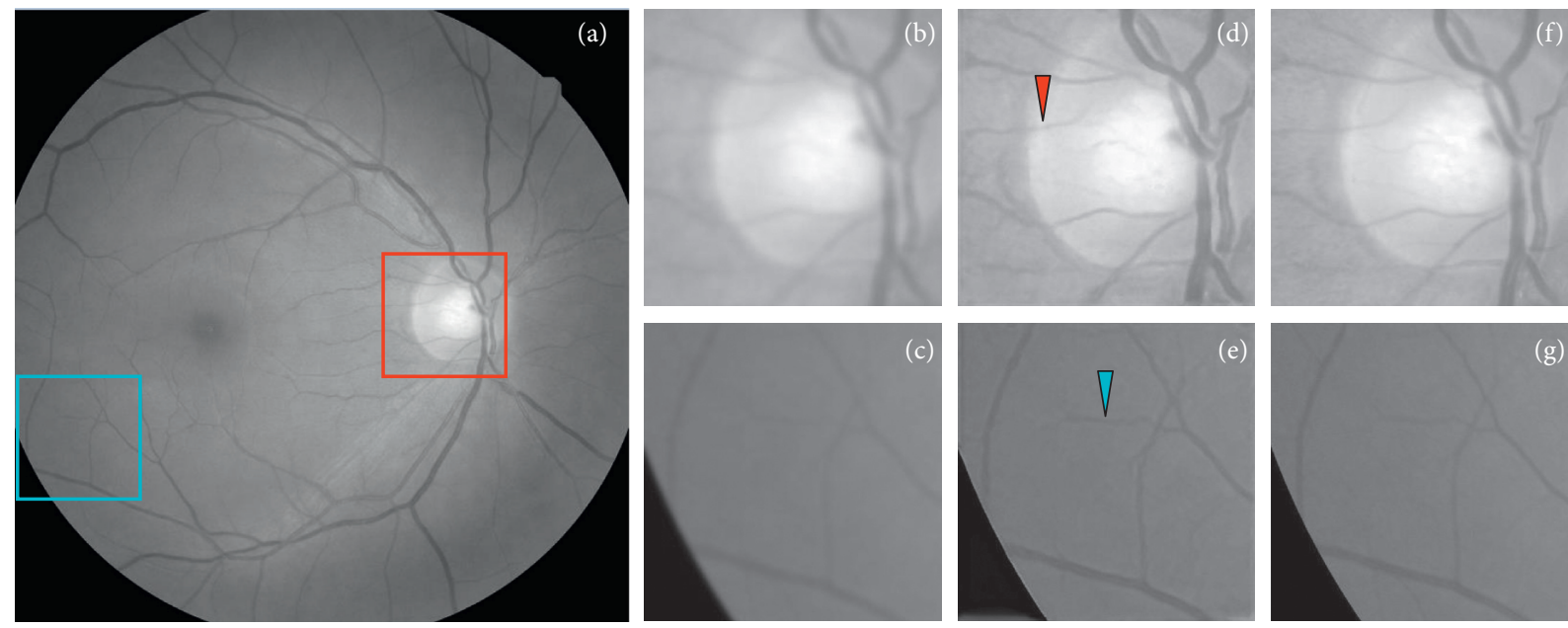

FIgURE 2: Experimental results on the simulated dataset. (a) Original uncropped image. (b, c) Blurry LR input. (d, e) The images processed by our method. (f, g) Ground truth. (b, d, f) Enlarged images of the red frame region enclosed in (a). (c, e, g) Enlarged images of the blue frame region enclosed in (a). Red arrow and blue arrow in (d) and (e) indicate the sharpened small blood vessels with our method.

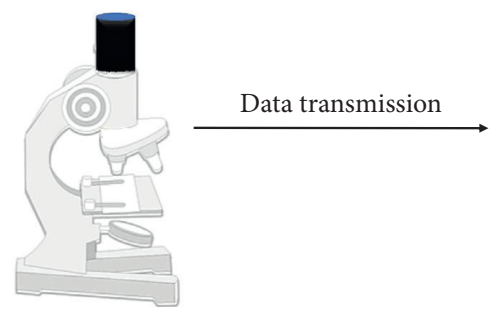

(a)

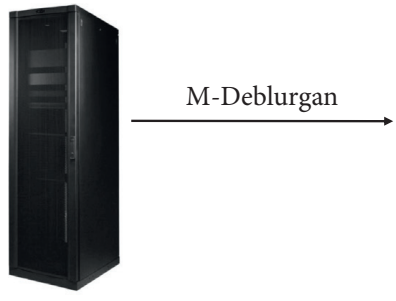

(b)

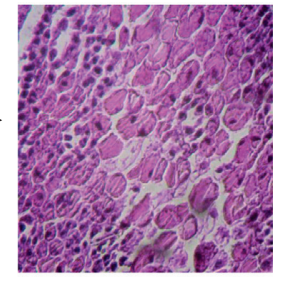

(c)

FIgURE 3: Experimental data acquisition and processing setup. (a) Optical microscope. (b) Computer. (c) Processed image.

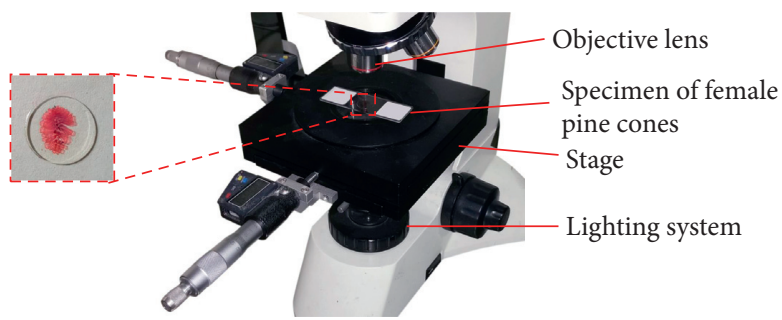

FIgURE 4: Experimental observation device.

network, the size of the input images is not limited. As shown in Figure 5, our proposed method can generate clearer images under real microscope observation scenes and has stronger robustness than other methods.

To measure the quantitative performance of the proposed network, we considered conventional indicators, namely, PSNR and SSIM. Table 1 is the quantitative comparison between the proposed method and other methods in the testing phase. The results show that the performance of the proposed network is superior to the existing methods in terms of PSNR and SSIM, with an increase of $4.8033 \mathrm{~dB}$ and $0.053 \mathrm{~dB}$ on the PINE CONES dataset. By introducing the optimal approximation module, the performance of the proposed network is greatly improved.
Comparing the U-net + SR model and our model in Figure 6, these two models performed similarly in the initial training stage. When the epoch is close to 200, the PSNR of M-Deblurgan is $2.7 \mathrm{~dB}$ higher than that of another model. The result demonstrates the superiority of the proposed model. Combining the data in Figure 7, it is not difficult to find that the proposed method has fewer parameters and higher PSNR values than other deep learning models. Fewer parameters will lead to less computer video memory consumption and improve the training efficiency of the model. Therefore, the M-Deblurgan is more robust. From the quantitative comparison, we can draw the conclusion that the M-Deblurgan based on the optimal approximation module is conducive to generating clearer and higher perceptual images. 

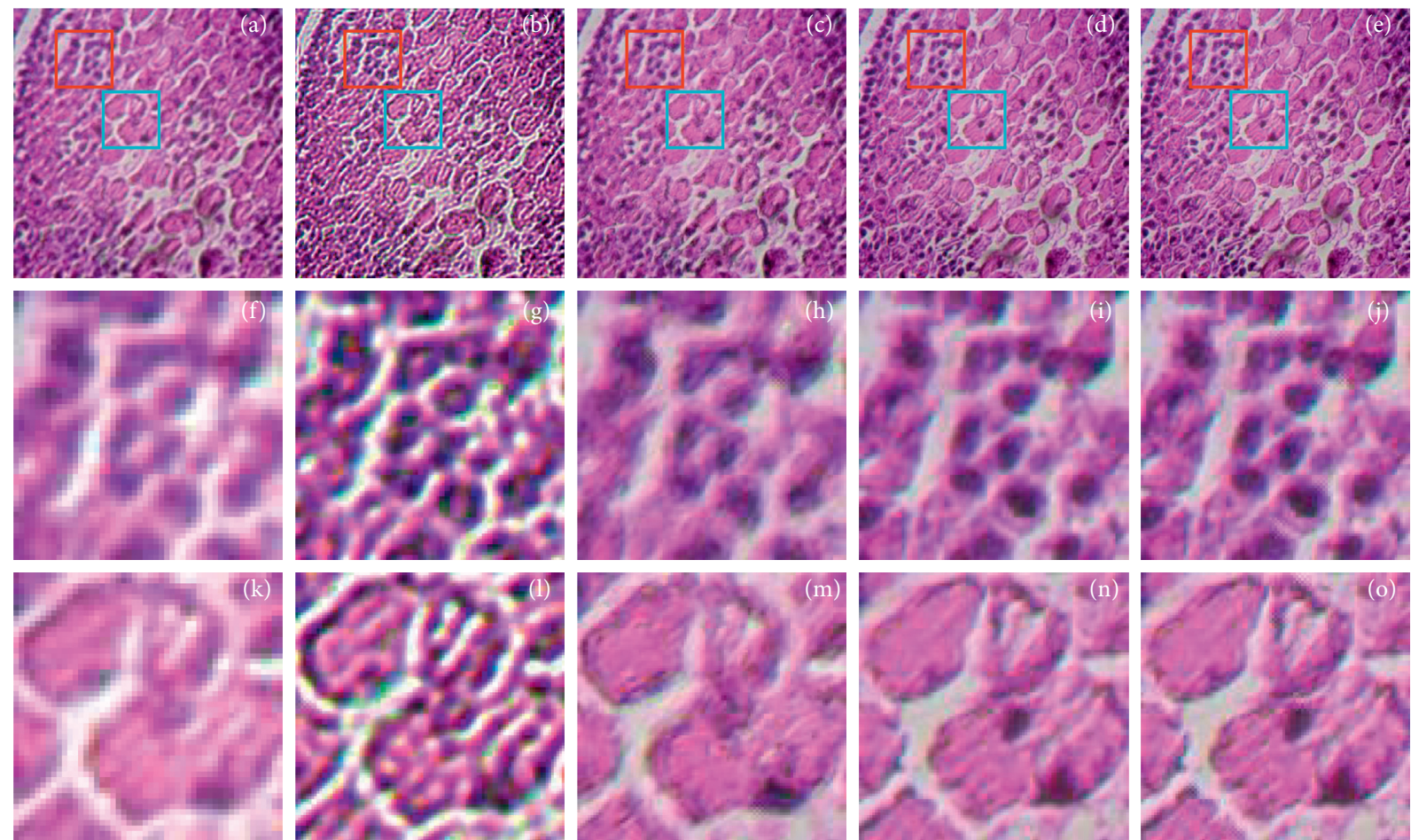

FIGURE 5: Experimental results on the PINE CONES dataset. (a) Image obtained by the $\times 5$ objective with the process of the defocused blur. (b-d) Processed image of (a) by the RL-Decov + SR, U-net + SR, and our method, respectively. (e) Images obtained by the $\times 10$ objective. $(\mathrm{f}-\mathrm{j}$ ) Enlarged images of the solid red region enclosed in (a-e), respectively. (k-o) Enlarged images of the solid blue region enclosed in (a-e), respectively.

TABLE 1: Quantitative comparison with other methods on the test dataset.

\begin{tabular}{lcccc}
\hline \multirow{2}{*}{ Method } & \multicolumn{2}{c}{ FIRE } & \multicolumn{2}{c}{ PINE CONES } \\
& PSNR & SSIM & PSNR & SSIM \\
\hline RL-Decov + SR & 26.1325 & 0.8034 & 25.2356 & 0.7822 \\
U-net + SR & 33.6004 & 0.9138 & 32.7293 & 0.9021 \\
Ours & 38.5014 & 0.9736 & 37.5326 & 0.9551 \\
\hline
\end{tabular}

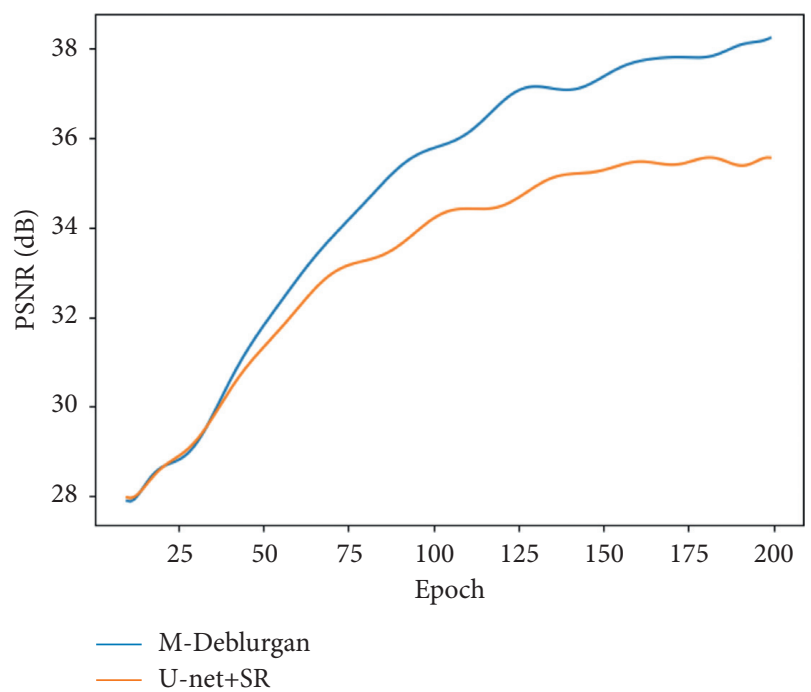

FIgURE 6: Quantitative analysis of two different deep learning methods on the training process.

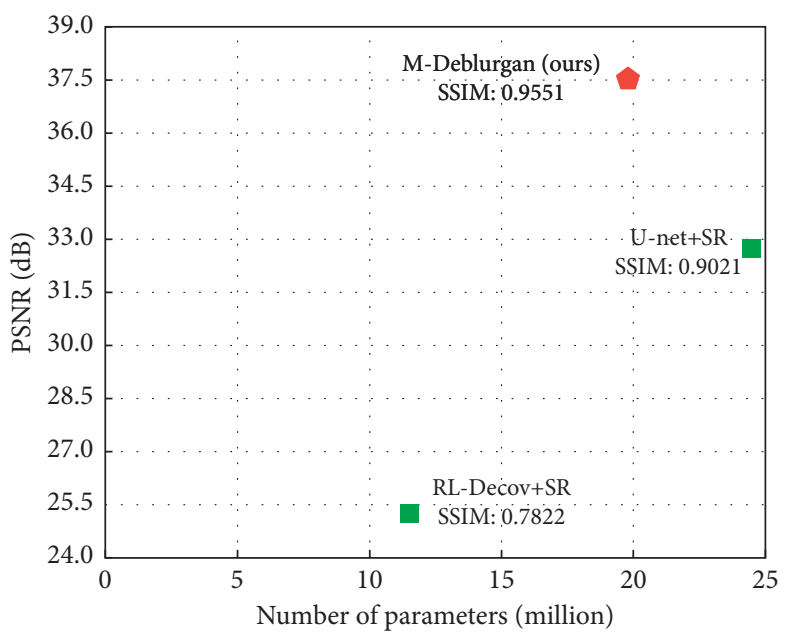

FIgURE 7: Comparison of PSNR and the model size of other methods on the PINE CONES dataset. The red pentagon is our method.

\section{Conclusion}

In this paper, we propose a deep learning model (MDeblurgan) to improve the imaging resolution and DOF of traditional optical microscopes. The M-Deblurgan doesn't require any manual manipulation of the PINE CONES dataset during the training process. Furthermore, the clear SR output can be obtained quickly when the blurry LR microscopic images are used as input. The experimental 
results show that our proposed method, especially the optimal approximation module, overcomes the limitations of the simple combination of deblurring and super-resolution tasks. The experiments show that the peak signal-to-noise ratio (PSNR) of our method can reach 37.5326 , and the structural similarity (SSIM) can reach 0.9551 in the experimental dataset. The experimental results show that the model is superior to existing methods in objective indicators such as visual quality and PSNR.

\section{Data Availability}

The data used to support the findings of this study are included within the article.

\section{Conflicts of Interest}

The authors declare that they have no conflicts of interest.

\section{Acknowledgments}

This work was supported by the National Natural Science Foundation of China (61975139 and 61927809).

\section{References}

[1] J. Cai, H. Zeng, H. Yong, Z. Cao, and L. Zhang, "Toward realworld single image super-resolution: a new benchmark and a new model," in Proceedings of the IEEE/CVF International Conference on Computer Vision, pp. 3086-3095, Seoul, South Korea, October 2019.

[2] Y. Huang, Z. Lu, Z. Shao et al., "Simultaneous denoising and super-resolution of optical coherence tomography images based on generative adversarial network," Optics Express, vol. 27, no. 9, pp. 12289-12307, 2019.

[3] Y. Kageyama, M. Isogawa, D. Iwai, and K. Sato, "ProDebNet: projector deblurring using a convolutional neural network," Optics Express, vol. 28, no. 14, pp. 20391-20403, 2020.

[4] O. Kupyn, V. Budzan, M. Mykhailych, D. Mishkin, and J. Matas, "Deblurgan: blind motion deblurring using conditional adversarial networks," in Proceedings of the IEEE Conference on Computer Vision and Pattern Recognition, pp. 8183-8192, Salt Lake City, UT, USA, June 2018.

[5] Z. Luo, A. Yurt, R. Stahl et al., "Pixel super-resolution for lensfree holographic microscopy using deep learning neural networks," Optics Express, vol. 27, no. 10, pp. 13581-13595, 2019.

[6] Z. Niu, J. Shi, L. Sun, Y. Zhu, J. Fan, and G. Zeng, "Photonlimited face image super-resolution based on deep learning," Optics Express, vol. 26, no. 18, pp. 22773-22782, 2018.

[7] W.-Z. Shao, Y.-Y. Liu, L.-Y. Ye et al., "DeblurGAN+: revisiting blind motion deblurring using conditional adversarial networks," Signal Processing, vol. 168, Article ID 107338, 2020.

[8] R. Strack, "Deep learning advances super-resolution imaging," Nature Methods, vol. 15, no. 6, p. 403, 2018.

[9] H. Sim and M. Kim, "A deep motion deblurring network based on per-pixel adaptive kernels with residual down-up and up-down modules," in Proceedings of the IEEE/CVF Conference on Computer Vision and Pattern Recognition Workshops, p. 0, Long Beach, CA, USA, June 2019.

[10] C. Belthangady and L. A. Royer, "Applications, promises, and pitfalls of deep learning for fluorescence image reconstruction," Nature Methods, vol. 16, no. 12, pp. 1215-1225, 2019.
[11] H. Wang, Y. Rivenson, Y. Jin et al., "Deep learning enables cross-modality super-resolution in fluorescence microscopy," Nature Methods, vol. 16, no. 1, pp. 103-110, 2019.

[12] M. Weigert, U. Schmidt, T. Boothe et al., "Content-aware image restoration: pushing the limits of fluorescence microscopy," Nature Methods, vol. 15, no. 12, pp. 1090-1097, 2018.

[13] O. Abdel-Hamid, A.-r. Mohamed, H. Jiang, L. Deng, G. Penn, and $\mathrm{D}$. $\mathrm{Yu}$, "Convolutional neural networks for speech recognition," IEEE/ACM Transactions on audio, speech, and language processing, vol. 22, no. 10, pp. 1533-1545, 2014.

[14] M. G. L. Gustafsson, "Surpassing the lateral resolution limit by a factor of two using structured illumination microscopy. short communication," Journal of Microscopy, vol. 198, no. 2, pp. $82-87,2000$.

[15] S. W. Hell and J. Wichmann, "Breaking the diffraction resolution limit by stimulated emission: stimulated-emissiondepletion fluorescence microscopy," Optics Letters, vol. 19, no. 11, pp. 780-782, 1994.

[16] S. Van de Linde, A. Löschberger, T. Klein et al., "Direct stochastic optical reconstruction microscopy with standard fluorescent probes," Nature Protocols, vol. 6, no. 7, pp. 991-1009, 2011.

[17] P. Sarder and A. Nehorai, "Deconvolution methods for 3-D fluorescence microscopy images," IEEE Signal Processing Magazine, vol. 23, no. 3, pp. 32-45, 2006.

[18] W. Ouyang, A. Aristov, M. Lelek, X. Hao, and C. Zimmer, "Deep learning massively accelerates super-resolution localization microscopy," Nature Biotechnology, vol. 36, no. 5, pp. 460-468, 2018.

[19] C. Qiao, D. Li, Y. Guo et al., "Evaluation and development of deep neural networks for image super-resolution in optical microscopy," Nature Methods, vol. 18, no. 2, pp. 194-202, 2021.

[20] H. Zhao, Z. Ke, N. Chen et al., “A new deep learning method for image deblurring in optical microscopic systems," Journal of Biophotonics, vol. 13, Article ID e201960147, 2020.

[21] I. J. Goodfellow, J. Pouget-Abadie, M. Mirza et al., "Generative adversarial networks," Advances in Neural Information Processing Systems, vol. 3, pp. 2672-2680, 2014.

[22] M. Ingaramo, A. G. York, E. Hoogendoorn, M. Postma, H. Shroff, and G. H. Patterson, "Richardson-lucy deconvolution as a general tool for combining images with complementary strengths," ChemPhysChem, vol. 15, no. 4, pp. 794-800, 2014.

[23] O. Ronneberger, P. Fischer, and T. Brox, "U-net: convolutional networks for biomedical image segmentation," in Medical Image Computing and Computer-Assisted Intervention, Pt Iii, Lecture Notes in Computer Science, N. Navab, J. Hornegger, W. M. Wells, and A. F. Frangi, Eds., Springer International Publishing Ag, New York, NY, USA, 2015. 